Finance Stochast. 1, 251-257 (1997)

$\overline{\text { Finance and }}$

Stochastics

(c) Springer-Verlag 1997

\title{
A note on the existence of unique equivalent martingale measures in a Markovian setting
}

\author{
Tina Hviid Rydberg ${ }^{\star}$ \\ University of Aarhus, Department of Theoretical Statistics, Ny Munkegade Bldg. 530, \\ DK-8000 Århus C, Denmark (e-mail: tina@mi.aau.dk)
}

\begin{abstract}
Simple sufficient conditions for the existence of a unique equivalent martingale measure are provided. Furthermore, these conditions give us a handle on situations where an equivalent martingale measure cannot exist. The existence of a unique equivalent martingale measure is of relevance to problems in mathematical finance. Two examples of models for which the question of existence was unresolved are studied. By means of our results existence of a unique equivalent measure up to an explosion time is proved.
\end{abstract}

Key words: Explosion time, hyperbolic diffusion processes, normal inverse Gaussian diffusion processes, stochastic differential equations, unique solution in law

JEL classification: G13

Mathematics Subject Classification (1991): 60G44, 60J60

\section{Introduction}

In mathematical finance and other areas of applied probability theory it is often important to determine when a certain equivalent martingale measure exists. In finance, it is of interest since the fair price of a derivative asset, such as a European or American call option, may be calculated as the mean value of the discounted payoff function under the unique equivalent martingale measure provided that this measure does in fact exist. Existence and uniqueness hold in particular for the Black-Scholes model.

* Financial support from Centre for Non-Linear Modelling in Economics, Department of Economics, University of Aarhus, is gratefully acknowledged.

Manuscript received: May 1996; final version received: March 1997 
In other, perhaps more realistic, models such as the hyperbolic diffusion model proposed by Bibby and Sørensen (1997) or the normal inverse Gaussian diffusion model proposed by Rydberg (1996), it may be difficult or perhaps even impossible to show existence by standard conditions such as the sufficient, but not necessary conditions proposed by Novikov and Kazamaki. The Novikov and Kazamaki conditions are discussed, for instance, in Revuz and Yor (1994); Chapter VIII. Here we will give an alternative method for showing the existence of a unique equivalent martingale measure in a Markovian setting. The method rests on proving uniqueness in law of solutions to stochastic differential equations (abbreviated to SDEs) without drift.

Section 2 contains the proposed theorem for existence of a unique equivalent martingale measure and a corollary. The uniqueness of the equivalent martingale measure is automatic since we require uniqueness of the solution to the SDE under the changed measure. The theorem furthermore gives us a handle on certain situations where an equivalent martingale measure cannot exist. This is discussed in connection to fair pricing in Remark 2.3.

Section 3 contains two examples of financial models where the question of existence of an equivalent martingale measure was unresolved. It was these models which motivated this paper. For these models the theorem from Sect. 2 implies existence of a unique equivalent measure only up to the first time zero is hit. From the theory we present it is also seen that an equivalent martingale measure cannot exist if the discounted price process leaves the interval, on which it should be defined, under the changed measure. This is what happens in the examples.

In Sect. 4 we briefly explain the "stopping" technique of Liptser and Shiryayev (1977) which is used in the proof of Theorem 2.1.

\section{Existence of a unique equivalent martingale measure}

Let $\sigma$ and $b$ be Borel-measurable functions $[0, T] \times I \mapsto I, I=(l, r) \subseteq \mathbf{R}$. Assume that $X$ is a solution of the SDE given by

$$
d X_{t}=b\left(t, X_{t}\right) d t+\sigma\left(t, X_{t}\right) d W_{t}, \quad X_{0}=x,
$$

where $W_{t}$ is a Wiener process defined on a probability space $(\Omega, \mathscr{F}, P)$ equipped with the filtration $\left\{\mathscr{F}_{t}\right\}_{0 \leq t \leq T}$. Throughout the paper $\mathscr{F}_{t}$ is the natural filtration generated by $X_{t}$.

Assumption 2.1. We assume in the sequel the existence of a solution to the SDE $\mathbf{e}_{x}(\sigma, b) . \mathbf{e}_{x}(\sigma, b)$ denotes the SDE problem starting in $x$ with coefficients $b$ and $\sigma$, see Revuz and Yor (1994); Chapter IX, for this notation. But here the state space is $I$.

Lemma 2.1. Let $I=\mathbf{R}$. Assume that Assumption 2.1 is satisfied, the coefficients are time-homogeneous and $\sigma^{2}>0$. Then there is existence and uniqueness in law for the $\operatorname{SDE} \mathbf{e}_{x}(\sigma, 0)$. 
Proof of Lemma 2.1: This is immediately seen using Zvonkin's observation (see e.g. Rogers and Williams (1987); Sect. V.8). More specifically, by putting (1) on its natural scale, thus removing the drift, using the scale function, we find that there exists a solution. Since $\sigma^{2}>0$, uniqueness follows from Engelbert and Schmidt (1984).

Let $\left(Y, W^{*}\right)\left(W^{*}\right.$ is a $P^{*}-$ Wiener process) be a solution to the $\operatorname{SDE} \mathbf{e}_{y}(\sigma, 0)$

$$
d Y_{t}=\sigma\left(t, Y_{t}\right) d W_{t}^{*}, \quad Y_{0}=y .
$$

Our goal is to determine when the distribution measures $\mu$ of $X$ and $\mu^{*}$ of $Y$ are equivalent.

The Girsanov theorem implies that the two laws $\mu$ and $\mu^{*}$ are equivalent on $[0, T]$ if the process given by

$$
N_{t}=\exp \left(-\frac{1}{2} \int_{0}^{t} \frac{b^{2}\left(s, X_{s}\right)}{\sigma^{2}\left(s, X_{s}\right)} d s-\int_{0}^{t} \frac{b\left(s, X_{s}\right)}{\sigma\left(s, X_{s}\right)} d W_{s}\right),
$$

is a martingale and the solution of the SDE given by equation (2) is unique in law. The process $N_{t}$ is always a supermartingale with $N_{0}=1$, and thus if $E^{P}\left(N_{T}\right)=1, N_{t}$ is in fact a martingale. To prove that $E^{P}\left(N_{T}\right)=1$ can be an extremely difficult task. The question of equivalence of measures has recently been addressed by Pedersen (1995) where a more general result in a martingale problem setting can be found.

We make the following assumption. The reason for this assumption will become clear in Sect. 4.

Assumption 2.2. From now on we assume that

$$
\int_{10, t]} \frac{b^{2}\left(s, u_{s}\right)}{\sigma^{2}\left(s, u_{s}\right)} d s<\infty \quad \mu, \mu^{*}-\text { a.s. on } C[0, T] \text { and for each } t<T \text {. }
$$

Remark 2.1. It should be noted that if Assumption 2.2 is fulfilled then the financial market described by the discounted price process $X$, which is a solution to $\operatorname{SDE} \mathbf{e}(\sigma, b)$, is complete (see Hansen (1996)) in the following sense: The self-financing trading strategies are well defined and the contingent claim $H$, $H \in \mathscr{T}_{T}, H>0$, such that $E^{P}\left[N_{T} H\right]<\infty$, is attainable at the fair price given by

$$
\pi(H)=E^{P}\left[N_{T} H\right] .
$$

If the unique equivalent martingale measure, $P^{*}$, exists then

$$
\pi(H)=E^{P}\left[N_{T} H\right]=E^{P^{*}}[H] .
$$

For further discussion of prices in financial models, see Remark 2.3. 
Theorem 2.1. Assume that there is existence and uniqueness in law to the SDE $\mathbf{e}_{y}(\sigma, 0)$ for every starting point $y \in I$ and that Assumptions 2.1 and 2.2 are fulfilled. Then the distribution measure $\mu^{*}$ of the solution to $\mathbf{e}_{y}(\sigma, 0)$ is equivalent to the distribution measure $\mu$ of the solution to $\mathbf{e}_{x}(\sigma, b)$ on $[0, T]$.

Remark 2.2. Using the notation $\mathbf{e}_{y}(\cdot, \cdot)$ above implies that the solution to $\mathbf{e}_{y}(\sigma, 0)$ has to live on $I$ specified by $\mathbf{e}_{x}(\sigma, b)$.

Proof of Theorem 2.1: To use the technique of Liptser and Shiryayev (1977), which will be presented in Sect. 4, all we need is a unique solution in law of the $\operatorname{SDE}$ given in (7) in Sect. 4. Since the $\operatorname{SDE} \mathbf{e}_{y}(\sigma, 0)$ is assumed to have a solution that is unique in law we also have that the corresponding martingale problem is well-posed. Due to the strong Markov property of the martingale problem it may be shown that the stopped martingale problem is also well-posed, see Jacod and Shiryaev (1987); Chapter III. Reverting to SDEs we now have that the stopped SDE in equation (7) has a solution that is unique in law. By the arguments in Sect. 4 it follows that $\mu$ and $\mu^{*}$ are equivalent.

Corollary 2.1. Let $I=\mathbf{R}$. Assume that the coefficients are time-homogeneous and $\sigma^{2}>0$. Furthermore, suppose that Assumptions 2.1 and 2.2 are fulfilled. Then the conditions in Theorem 2.1 are satisfied.

Proof of Corollary 2.1: This is a consequence of Lemma 2.1.

Remark 2.3. Assume that $b$ and $\sigma$ are $\mathrm{C}^{1}$ and $\sigma^{2}(x)>0$ for all $x \in I$, then for $x \in I$ the $\operatorname{SDE} \mathbf{e}_{x}(\sigma, b)$ has a unique solution $X^{x}$ up to the explosion time $e^{*}=\lim _{n \uparrow \infty} \tau_{n}$ where $\tau_{n}=\inf \left\{t: X^{x} \notin\left[a_{n} ; b_{n}\right]\right\}$ and where $a_{n}$ and $b_{n}$ $(n=1,2, \ldots)$ are chosen such that $l<a_{n}<b_{n}<r$ and $a_{n} \downarrow l$ and $b_{n} \uparrow r$ (Ikeda and Watanabe (1989); p. 446). We always assume that there exists a nonexploding solution to $\mathbf{e}_{x}(\sigma, b)$, so if the solution to $\mathbf{e}_{x}(\sigma, 0)$ explodes there cannot exist a unique equivalent martingale measure in the usual sense. We do have that $P_{\mid \mathscr{T}_{\tau_{n}}} \sim P_{\mid \mathscr{T}_{\tau_{n}}}^{*}$ but not $P_{\mid \mathscr{T}_{e}^{*}} \sim P_{\mid \mathscr{F}_{e}^{*}}^{*}$. This implies that

$$
E^{P}\left[N_{T} H\right]=E^{P}\left[\mathbf{1}_{\left\{e^{*}>T\right\}} N_{T} H\right]+E^{P}\left[\mathbf{1}_{\left\{e^{*} \leq T\right\}} N_{T} H\right]
$$

and hence

$$
E^{P}\left[N_{T} H\right]=E^{P^{*}}\left[\mathbf{1}_{\left\{e^{*}>T\right\}} H\right],
$$

i.e. with the definition of a fair price given in Remark 2.1, the price of the contingent claim $H=1$ would have a price smaller than 1 since

$$
E^{P^{*}}\left[\mathbf{1}_{\left\{e^{*}>T\right\}}\right]=P^{*}\left(e^{*}>T\right) \leq 1 .
$$

The 3-dimensional Bessel process, $\sigma=1$ and $b(x)={ }_{x}^{1}$, given as an example in Delbaen and Schachermayer (1995), is a nice example of a situation where the solution of $\mathbf{e}_{x}(\sigma, b)$ lies in $\mathbf{R}_{+}$, i.e. $I=\mathbf{R}_{+}$, but there is no solution of $\mathbf{e}_{x}(\sigma, 0)$ on $\mathbf{R}_{+}$since this is a Brownian motion which lives on $\mathbf{R}$. 


\section{Motivation}

As mentioned in the introduction it can be rather difficult to decide whether a unique equivalent martingale measure exists or not. In particular, this has been the case for the hyperbolic diffusion model proposed by Bibby and Sørensen (1997) and the normal inverse Gaussian diffusion model proposed by Rydberg (1996) which are special cases of the generalized hyperbolic diffusion models, see Rydberg (1996). The hyperbolic diffusion model is studied in relation to Danish stock price data in Bibby and Sørensen (1997) and in Rydberg (1996), the normal inverse Gaussian diffusion model is used to model the log returns of US stock prices. Both models give very good fits to observed data and in Rydberg (1996), it is seen that the normal inverse Gaussian diffusion model is capable of explaining the so called "smile".

The models can be characterized in the following way. Let

$$
Z_{t}=\int_{0}^{t} \sigma\left(Z_{s}\right) d W_{s}
$$

The generalized hyperbolic diffusion processes $Z_{t}$ now appear as a result of different specifications of the function $\sigma$. The hyperbolic diffusion model is the special case where

$$
\sigma(z)=\kappa \exp \left(\frac{1}{2}\left(\alpha \sqrt{ } \delta^{2}+(z-\mu)^{2}-\beta(z-\mu)\right)\right),
$$

and the normal inverse Gaussian diffusion model is the special case where

$$
\sigma(z)=\kappa \exp \left(-\frac{1}{2} \beta(z-\mu)\right) \sqrt{\frac{\sqrt{ } \delta^{2}+(z-\mu)^{2}}{K_{1}\left(\alpha \sqrt{ } \delta^{2}+(z-\mu)^{2}\right)}} .
$$

Furthermore, the parameters fulfill

$$
\kappa>0, \mu \in \mathbf{R}, \delta>0,0 \leq|\beta|<\alpha
$$

and $K_{1}$ is the modified Bessel function of the third kind with index 1 .

The generalized hyperbolic diffusion processes are constructed such that they are ergodic with a generalized hyperbolic distribution as the stationary distribution. For these processes the $\sigma$ functions are continuous and $\sigma^{2}>0$. Therefore we see that the SDE in equation (3) has a unique solution in law for every starting point $z$.

Now, if we consider

$$
S_{t}=S_{0} \exp \left(Z_{t}\right)
$$

as a stock price model then, according to Theorem 2.1, we need to show that the SDE, given by

$$
S_{t}^{*}=\sigma\left(\log \left(S_{t}^{*}\right)\right) S_{t}^{*} d W_{t}^{*}
$$

also has a unique solution on $\mathbf{R}_{+}$. Unfortunately, $S^{*}$ explodes downwards and therefore there cannot exist an equivalent martingale measure, but only an equivalent measure up to $e^{*}$, the explosion time. 


\section{The "stopping" technique}

The technique of Liptser and Shiryayev (1977) is based on stopped processes. For $n \in\{1,2, \ldots\}$, let the stopping time $\tau_{n}(y)$ be defined by (see e.g. Liptser and Shiryayev (1977); Chapter 6)

$$
\tau_{n}(y)=\inf \left\{t \in[0, T] \mid \int_{] 0, t]} \frac{b^{2}\left(s, y_{s}\right)}{\sigma^{2}\left(s, y_{s}\right)} d s \geq n\right\}, \quad \inf \{\emptyset\}=T .
$$

The stopping time is a function $C[0, T] \mapsto \mathbf{R}_{+}$, where $C[0, T]$ denotes the space of continuous paths on $[0, T]$. This definition of $\tau_{n}$ immediately gives us that

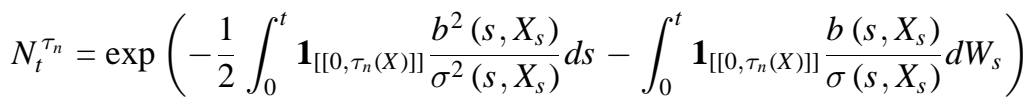

has mean value equal to 1 since the Novikov condition is now trivially fulfilled. The corresponding stopped processes $X^{\tau_{n}(X)}$ and $Y^{\tau_{n}(Y)}$ of (1) and (2) solve the SDEs given by

$$
\begin{aligned}
& d X_{t}^{\tau_{n}(X)}=\mathbf{1}_{\left[\left[0, \tau_{n}(X)\right]\right]} b\left(t, X_{t}^{\tau_{n}(X)}\right) d t+\mathbf{1}_{\left[\left[0, \tau_{n}(X)\right]\right]} \sigma\left(t, X_{t}^{\tau_{n}(X)}\right) d W_{t}, \\
& d Y_{t}^{\tau_{n}(Y)}=\mathbf{1}_{\left[\left[0, \tau_{n}(Y)\right]\right]} \sigma\left(t, Y_{t}^{\tau_{n}(Y)}\right) d W_{t}^{*} .
\end{aligned}
$$

If $\tau_{n}$ is a localization under both $P$ and $P^{*}$ then equivalence of the restricted measures $\mu_{\mid \mathscr{F}_{\tau_{n}}}$ and $\mu_{\mid \mathscr{F}_{\tau_{n}}}^{*}$ implies that $\mu \sim \mu^{*}$ on $[0, T]$.

Remark 4.1. If $b$ and $\sigma$ are continuous and $\sigma^{2}>0$, then $\tau_{n}$ is a localization.

However, all we need for $\tau_{n}$ to be a localization is Assumption 2.2.

\section{Concluding remarks}

A new theorem for the existence of a unique equivalent martingale measure was presented. It was also noted that we got a handle on situations where an equivalent martingale measure cannot exist. The examples in Sect. 3 showed that if we are in a Markovian setting Theorem 2.1 gives an easy way to check if an equivalent martingale measure exists or not.

The version of Theorem 2.1 is for functions $\sigma:[0, T] \times \mathbf{R} \mapsto \mathbf{R}$ and $b$ : $[0, T] \times \mathbf{R} \mapsto \mathbf{R}$. A generalization of Theorem 2.1 to functions on $[0, T] \times \mathbf{R}^{d}$ is straightforward. But Lemma 2.1 and Corollary 2.1 do not extend to the multidimensional case, at least not in any immediate way, for they use specific results, concerning scale functions, etc., which only have meaning for one-dimensional diffusions. Theorem 2.1 is easily generalized to situations where equivalence of the distribution measures of $\mathbf{e}_{x}\left(\sigma, b_{1}\right)$ and $\mathbf{e}_{x}\left(\sigma, b_{2}\right)$ are under consideration. This is done by changing Assumption 2.2 into 


$$
\begin{aligned}
& \int_{] 0, t]} \frac{\left(b_{2}\left(s, u_{s}\right)-b_{1}\left(s, u_{s}\right)\right)^{2}}{\sigma^{2}\left(s, u_{s}\right)} d s<\infty \\
& \mu, \mu^{*}-\text { a.s. on } C[0, T] \text { and for each } t<T \text {. }
\end{aligned}
$$

The results in Sect. 2 are formulated for $t \in[0, T]$ since we are only interested in pricing contingent claims $H \in \mathscr{T}_{T}$. The results extend to $\mathbf{R}_{+}$with minor amendments.

Acknowledgements. Thanks are due to S. E. Graversen, J. Pedersen, M. Sørensen, J. A. Nielsen and O. E. Barndorff-Nielsen for fruitful discussions and comments. The author is also grateful to the associate editor for constructive comments and to an anonymous referee for drawing attention to the Delbaen and Schachermayer paper and the examples therein.

\section{References}

Bibby, B.M., Sørensen, M.: A Hyperbolic Diffusion Model for Stock Prices. Finance Stochast. 1, 25-41 (1997)

Delbaen, F., Schachermayer, W.: Arbitrage possibilities in Bessel processes and their relations to local martingales. Probab. Theory Relat. Fields 102, 357-366 (1995)

Engelbert, H.J., Schmidt, W.: On One-Dimensional Stochastic Differential Equations with Generalized Drift. In: M. Metivier, E. Pardoux (eds.) Lecture Notes on Control and Information Science, Vol. 69, pp. 143-155. Proceedings of the IFIP-WG 7/1 Working Conference on Stochastic Differential Systems, Marseille-Luminy, France, March 12-17, 1984. Berlin, Heidelberg, New York: Springer 1984

Hansen, A.T.: Complete Market Pricing in the Wiener-Filtration without Existence of a Martingale Measure, Department of Operations Research, Institute of Mathematics, University of Århus 1996

Ikeda, N., Watanabe, S.: Stochastic Differential Equations and Diffusion Processes. Mathematical Library, Vol. 24, 2nd edn. Amsterdam: North-Holland 1989

Jacod, J., Shiryaev, A.N.: Limit Theorems for Stochastic Processes. Grundlehren der mathematischen Wissenschaften, Vol. 288. Berlin, Heidelberg, New York: Springer 1987

Liptser, R.S., Shiryayev, A.N.: Statistics of Random Processes I - General Theory. Applications of Mathematics, Vol. 5. Berlin, Heidelberg, New York: Springer 1997

Pedersen, J.: Two Application of Stopped Martingale Problems, Research Report 320, Department of Theoretical Statistics, Institute of Mathematics, University of Århus, 1995

Revuz, D., Yor, M.: Continuous Martingales and Brownian Motion. Grundlehren der mathematischen Wissenschaften, Vol. 293, 2nd edn. Berlin, Heidelberg, New York: Springer 1974

Rogers, L.C.G., Williams, D.: Diffusions, Markov Processes and Martingales: Itô Calculus, Vol. 2. Chichester: John Wiley \& Sons 1987

Rydberg, T.H.: Generalized Hyperbolic Diffusions with Applications Towards Finance, Research Report 342, Department of Theoretical Statistics, Institute of Mathematics, University of Århus, 1996 